\title{
EDITORIAL
}

\section{Autophagy: Eat Yourself to Live}

Autophagy is a metabolic process of consumption of the body's own tissues occurring in starvation and certain diseases. The field of autophagy has grown enormously over the past 10-15 years, with rapid advances in our understanding of the regulatory mechanisms that control autophagy pathways in mammalian systems. It has also improved our understanding of the physiological influences of autophagy in health and disease. It all started in the mid1950s when Sam Clark Jr. of the School of Medicine at Washington University in St. Louis looked through his electron microscope at newborn mouse kidneys and used the term cytolysome for the membrane-bound structures within the cytoplasm of the kidney cells. He and his colleague Edward Essner wrote in 1962 that "Within these cytolysomes remarkable events are in progress", "Cytoplasm has somehow found its way inside the droplets and is apparently in the process of digestion." These were the first descriptions of what is known today as macroautophagy and now referred to as autophagy.

The significance of autophagy in physiology and medicine was fully documented by Yoshinori Ohsumi's research in the 1990's and because of his innovative effort, autophagy is now acknowledged as a central process in cell physiology with foremost implications for human disease and health. It is for this discovery that he was awarded the 2016 Nobel Prize in physiology or medicine.

The contribution of autophagy to cellular health under both normal and stress conditions implies important physiological and pathological roles for this tightly regulated and precisely orchestrated process. Today, autophagy is recognized as a critical process for maintaining cellular homeostasis, as well as for responding to stressors, such as nutrient deficiency, which may potentially compromise cell survival. Autophagy can promote degradation en masse for a large number and variety of substrates by delivering them to lysosomes, enabling cells to quickly and efficiently generate recycled basic building materials in the face of a wide range of nutritional deficiencies. It plays a housekeeping role in removing misfolded or aggregated proteins, clearing damaged organelles, such as mitochondria, endoplasmic reticulum and peroxisomes, as well as eliminating intracellular pathogens either randomly or in a targeted fashion. Thus, autophagy is generally thought of as a survival mechanism, although its deregulation has been linked to non-apoptotic cell death.

Autophagy has opposing, context-dependent roles in cancer. In contrast to the apparent role of autophagy in blocking tumor initiation, however, there is evidence that autophagy can also promote malignancy in established tumors. This has led to the therapeutic targeting of autophagy in cancer. Recent research has revealed that the role of autophagy in promoting tumor progression is not limited to cellular effects in the cancer itself, but extends to modulation of the tumor microenvironment. Now time has begun to explore the function of autophagy in cancer metastasis, which is of particular interest given the dearth of effective therapeutic options for metastatic disease.

Recent research has discovered that autophagy is a critical modulator of a wide range of diseases and disorders, like Crohn's disease, heart conditions, PD etc. but the number of available chemical modulators of autophagy is still limited. The better understanding of the contribution of autophagy to disease initiation and progression should help to develop effective interventions targeting autophagy for the treatment of disease in the near future.

As a scientific field, the study of autophagy is at a very exciting juncture. Although researchers have partially uncovered some of the mechanisms related to autophagy initiation, progression, and regulation; our understanding of the role of autophagy in development and disease is at an early stage and there are still more questions than answers concerning the functions of the autophagy-related proteins. Further realization of the role of the process in pathophysiology promises to reveal as yet unanticipated avenues for therapeutic intervention.

We already knew that we must eat to live, and it's now clear that this can sometimes extend to eating ourselvesat least on a cellular level.

Dr. Zafar Amin shah 